\title{
Ice Roughness in Short Duration SLD Icing Events
}

\author{
Stephen T. McClain, ${ }^{1}$ Dana Reed, ${ }^{2}$ \\ Baylor University, Waco, TX, 76798-7356 \\ Mario Vargas, ${ }^{3}$ Richard E. Kreeger, ${ }^{4}$ \\ NASA Glenn Research Center, Cleveland, $\mathrm{OH} 44135$ \\ and \\ Jen-Ching Tsao ${ }^{5}$ \\ Ohio Aerospace Institute, Cleveland, $\mathrm{OH}, 44142$
}

\begin{abstract}
Ice accretion codes depend on models of roughness parameters to account for the enhanced heat transfer during the ice accretion process. While mitigating supercooled large droplet (SLD or Appendix O) icing is a significant concern for manufacturers seeking future vehicle certification due to the pending regulation, historical ice roughness studies have been performed using Appendix $\mathrm{C}$ icing clouds which exhibit mean volumetric diameters (MVD) much smaller than SLD clouds. Further, the historical studies of roughness focused on extracting parametric representations of ice roughness using multiple images of roughness elements. In this study, the ice roughness developed on a 21-in. NACA 0012 at $0^{\circ}$ angle of attack exposed to short duration SLD icing events was measured in the Icing Research Tunnel at the NASA Glenn Research Center. The MVD's used in the study ranged from 100 $\mu \mathrm{m}$ to $200 \mu \mathrm{m}$, in a $67 \mathrm{~m} / \mathrm{s}$ flow, with liquid water contents of either $0.6 \mathrm{gm} / \mathrm{m}^{3}$ or $0.75 \mathrm{gm} / \mathrm{m}^{3}$. The ice surfaces were measured using a Romer Absolute Arm laser scanning system. The roughness associated with each surface point cloud was measured using the two-dimensional self-organizing map approach developed by McClain and Kreeger (2013) resulting in statistical descriptions of the ice roughness.
\end{abstract}

\section{Nomenclature}

$\begin{array}{ll}\text { Ac } & =\text { accumulation parameter } \\ \text { AOA } & =\text { angle of attack } \\ b & =\text { codebook vectors } \\ h(i, j) & =\text { neighborhood function of } i \text { to } j \text { codebook vectors } \\ j & =\text { codebook vector index } \\ \mathrm{LWC} & =\text { liquid water content }\left[\mathrm{gm} / \mathrm{m}^{3}\right] \\ M & =\text { number of codebook vectors } \\ \mathrm{MVD} & =\text { median volumetric diameter }[\mu \mathrm{m}] \\ N & =\text { airfoil or mean ice shape surface normal coordinate direction } \\ R^{2} & =\text { coefficient of determination (regression) } \\ R^{d} & =\text { high-dimensional data space } \\ R M H & =\text { roughness maximum height } \\ R_{q} & =\text { the root-mean-square or "standard deviation" roughness height } \\ r_{a} & =\text { leading edge radius of curvature } \\ \mathrm{SOM} & =\text { Self-Organizing Map }\end{array}$

\footnotetext{
${ }^{1}$ Associate Professor, Department of Mechanical Engineering, One Bear Place \#97356, and AIAA Senior Member.

${ }^{2}$ Undergraduate Student, Department of Mechanical Engineering, One Bear Place \#97356, and non-member.

${ }^{3}$ Aerospace Engineer, Icing Branch, 21000 Brookpark Rd., AIAA Associate Fellow.

${ }^{4}$ Aerospace Engineer, Icing Branch, 21000 Brookpark Rd., AIAA Associate Fellow.

${ }^{5}$ Principal Research Scientist, Icing Branch, 21000 Brookpark Rd., MS 11-2, AIAA Associate Fellow.
} 


$\begin{array}{ll}S & =\text { airfoil or mean ice shape surface tangential coordinate direction } \\ \text { SEE } & =\text { Standard error of the estimate for regression } \\ x & =\text { element of data set } \\ \alpha & =\text { local direction angle of manifold through a codebook vector } \\ \beta & =\text { manifold } \\ \gamma & =\text { direction angle of surface point relative to manifold direction through winning codebook vector } \\ \delta & =\text { scaling parameter governing neighborhood size } \\ \eta & =\text { learning rate }\end{array}$

\section{Introduction}

$\mathrm{T}$ HE effects of roughness on ice shapes are well documented. Ice roughness increases the skin friction drag, increases the rate of heat transfer from the freezing water layer to the airstream, and induces higher levels of turbulence in the airflow than would be observed on mean representative ice shapes without roughness. Further, ice roughness increases the droplet capture efficiency of the local surface relative to a smooth surface.

Capturing the enhanced convective heat transfer caused by the ice roughness is a critical aspect in modeling the accretion process. Since the freezing process is exothermic, energy must be transferred from the freezing front to the airstream. The local rate at which energy is transferred governs the rate of ice accumulation. Consequently, the regions with the highest convection coefficients are expected to exhibit the highest rates of ice shape growth.

While ice roughness and its geometric properties have been recognized as critical to the ice accretion process and to the reduction in iced aircraft performance in icing conditions, describing the nature of ice accretion roughness is not trivial. Classical studies of ice roughness during short-duration icing events include Anderson and Shin [1], Anderson et al. [2], and Shin [3]. For the afore mentioned measurements, images were acquired of the ice surface from multiple directions. Image analysis techniques were then used to determine the dimensions of each roughness element and the spacing to its nearest neighboring roughness element. The methods used in the classical studies were extremely laborious in terms of manual identification of each roughness element, use of the image analysis equipment, and identification of the roughness element in multiple images. Further, the results of the characterizations were morphological or deterministic, relating to individual bead height and width, as opposed to the statistical representations commonly used to describe randomly rough surfaces.

The historical investigations of ice roughness also focused on what is known as Appendix $\mathrm{C}$ roughness. Appendix $\mathrm{C}$ icing represents stratiform and cumuliform icing conditions that exhibit supercooled or partially frozen droplets with an MVD of 1-50 $\mu \mathrm{m}$ [4]. While Appendix C conditions were the primary focus of ice protection system certification in the past, supercooled large droplet or "Appendix O" conditions have become a significant issue regarding future vehicle and ice protection system certification [5]. While Appendix O conditions have MVDs much larger than Appendix C conditions, 50-1000 $\mu \mathrm{m}$, the trends for ice roughness development from the historical studies of Appendix $\mathrm{C}$ conditions are often extrapolated to Appendix $\mathrm{O}$ conditions when using ice accretion codes such as LEWICE [6].

In addition to changing certification requirements, recent improvements in laser scanning approaches have the potential to revolutionize the process of ice accretion shape characterization and surface roughness evaluation. Lee et al. [7] recently demonstrated measurements of different ice shapes formed in the NASA Icing Research Tunnel made using a Romer Absolute Arm laser scanning system. While laser scanning represents a tremendous leap in ice shape and roughness measurement ability, laser scans of ice shapes or castings of ice shapes present challenges. First, the scans typically result in point densities of 5000 points per $\mathrm{cm}^{2}$ of scanned surface area. For the leading edges of two-dimensional wings (airfoils) used in the NASA icing tunnel, which have 18 to $21 \mathrm{in}$. chord lengths, ice shape scans with a $10-\mathrm{cm}$ spanwise width typically result in more than 1 million measurement points. Secondly, on two-dimensional airfoils, horn structures and the geometrically closed-loop nature of airfoils lead to multi-valued functions describing the airfoil surface. The multi-valued function description of airfoils with complex horn shapes results in situations where standard regression cannot be employed to describe the entire airfoil at once. To evaluate the roughness along an iced airfoil, the mean ice-shape or ice form must be extracted. Thirdly, the radii of curvature of horn structures may be on the same order of magnitude as the roughness heights for long-accretion time ice shapes. The comparable sizes of horn structures and roughness creates difficulties in discerning the difference between ice form changes and roughness features. Finally, most ice shapes, even on straight wings, have considerable three-dimensional variations along the span of the wing.

Clustering methods for non-linear manifold detection enable a rigorous evaluation of ice shape roughness. McClain et al. [8] applied the self-organizing map (SOM) approach to characterize the nature of airfoils with rime 
ice, glaze ice, and multi-horn ice shapes. The primary objective of McClain et al. [8] was to demonstrate how the SOM could be used not only to capture the mean ice form or shape, but to expand the SOM approach using multidimensional statistics to characterize the ice-surface coverage limits. McClain and Kreeger [9] revisited the SOM approach and demonstrated a method to unwrap the point cloud using SOM representations and use the distribution of the surface points closest to each SOM codebook vector to evaluate the local surface roughness.

The objective of this effort was to characterize SLD ice roughness in short duration icing events using the Romer laser scanner and employing the approach of McClain and Kreeger [9] to create statistical descriptions of the ice roughness. In all of the cases, the icing events were ended before enough ice was accumulated on the airfoil surfaces to substantially alter the aerodynamic performance. The primary aims of the effort were 1) to characterize how SLD roughness develops with increasing accumulation parameter (indicating longer accretion times) given fixed cloud properties and 2) to characterize the influence of the MVD on ice roughness provided equivalent accumulation parameters.

\section{Experimental Method}

All of the experimental measurements were performed in the Icing Research Tunnel (IRT) at NASA Glenn Research Center in Cleveland, OH. A straight, 21 -in. $(53.3-\mathrm{cm})$ NACA 0012 airfoil, which spanned the entire 72 -in. $(182.9 \mathrm{~cm})$ test section, was used for the study. The test airfoil was set to $0^{\circ} \mathrm{AOA}$ for all cases.

With the exception of the MVD values, the conditions used for the study were based on the conditions employed in the historical literature for the Appendix C cases [1]-[3]. All cases were run with a nominal freestream velocity of $129.7 \mathrm{knots}(66.723 \mathrm{~m} / \mathrm{s})$. Table 1 presents the freestream total temperature $\left(T_{\text {total }}\right)$, the frestream velocity, the median volumetric diameter, the liquid water content, the accumulation parameter and the freezing fraction. The accumulation parameter is a derived quantity calculated using

$$
A_{c}=\frac{L W C \cdot V \cdot \Delta t_{s}}{2 \cdot r_{a} \cdot \rho_{\text {ice }}}
$$

Where $r_{a}$ is the leading-edge radius of curvature of the NACA 0012 airfoil, and $\rho_{\text {ice }}$ is the density of ice at the freestream temperature.

Table 1. Cases Used for the SLD Roughness Characterizations

\begin{tabular}{|c|c|c|c|c|c|c|c|}
\hline Run Number & $\begin{array}{c}T_{\text {total }} \\
\left({ }^{\circ} \mathrm{F}\right)\end{array}$ & $\begin{array}{c}V \\
(\mathrm{knots})\end{array}$ & $\begin{array}{c}\mathrm{MVD} \\
(\mu \mathrm{m})\end{array}$ & $\begin{array}{c}\mathrm{LWC} \\
\left(\mathrm{gm} / \mathrm{m}^{3}\right)\end{array}$ & $\begin{array}{c}\Delta t_{s} \\
(\mathrm{~s})\end{array}$ & $\begin{array}{c}\text { Accumulation } \\
\text { Parameter }\end{array}$ & $\begin{array}{c}\mathrm{n} \\
\text { Freezing } \\
\text { Fraction }\end{array}$ \\
\hline 112912.04 & 27.8 & 129.7 & 100.0 & 0.60 & 27 & 0.0703 & 0.217 \\
\hline 112912.03 & 27.8 & 129.7 & 100.0 & 0.60 & 43 & 0.1120 & 0.217 \\
\hline 112912.02 & 27.8 & 129.7 & 100.0 & 0.60 & 55 & 0.1432 & 0.217 \\
\hline 112912.01 & 27.8 & 129.7 & 100.0 & 0.60 & 75 & 0.1953 & 0.217 \\
\hline 112812.07 & 27.8 & 129.7 & 100.0 & 0.60 & 94 & 0.2448 & 0.217 \\
\hline 113012.06 & 27.7 & 129.7 & 150.0 & 0.60 & 27 & 0.0703 & 0.217 \\
\hline 113012.05 & 27.7 & 129.7 & 150.0 & 0.60 & 43 & 0.1120 & 0.217 \\
\hline 113012.04 & 27.7 & 129.7 & 150.0 & 0.60 & 75 & 0.1953 & 0.217 \\
\hline 112912.06 & 27.7 & 129.7 & 150.0 & 0.60 & 94 & 0.2448 & 0.217 \\
\hline 112912.08 & 26.5 & 129.7 & 200.0 & 0.75 & 19 & 0.0618 & 0.217 \\
\hline 113012.01 & 26.5 & 129.7 & 200.0 & 0.75 & 27 & 0.0878 & 0.217 \\
\hline 112912.09 & 26.5 & 129.7 & 200.0 & 0.75 & 43 & 0.1398 & 0.217 \\
\hline 112912.10 & 26.5 & 129.7 & 200.0 & 0.75 & 75 & 0.2439 & 0.217 \\
\hline 112912.07 & 26.5 & 129.7 & 200.0 & 0.75 & 94 & 0.3057 & 0.217 \\
\hline
\end{tabular}

Table 1 shows that for the $200-\mu \mathrm{m}$ cases, a different liquid water content was used than for the $100-\mu \mathrm{m}$ and 150 $\mu \mathrm{m}$ cases. It was desired to use an LWC of $0.6 \mathrm{gm} / \mathrm{m}^{3}$ for all cases. However, a $200-\mu \mathrm{m}$ cloud with an LWC of 0.6 $\mathrm{gm} / \mathrm{m}^{3}$ case was outside the calibration envelope of the spray-bar system. Consequently, the $200-\mu \mathrm{m}$ cases were run at $0.75 \mathrm{gm} / \mathrm{m}^{3}$ and the spray times were shortened to match accumulation parameters. 
For each test, the airspeed and freestream total temperature were set and the spray bar pressures were selected to provide the appropriate LWC, MVD, and freezing fraction. A thermocouple embedded in the NACA 0012 airfoil was used to determine when the airfoil had reached thermal equilibrium with the flow. Once the thermocouple reported the static temperature of the flow indicating that it was in thermal equilibrium with the flow, the spray bars were actuated and closed after the predetermined spray time.

Following the completion of the icing spray, the wind tunnel velocity was reduced to approximately 10 knots while keeping the static temperature around $-4{ }^{\circ} \mathrm{C}$ to avoid thawing of the ice shape. The iced airfoil was painted using an alcohol-based titanium dioxide paint. The Romer Absolute Arm was placed in the IRT test section upstream of the airfoil. A scan was then made of the leading 120-150 mm of the airfoil leading edge. The scans were approximately $100-\mathrm{cm}$ wide (in the spanwise direction) and were performed at the center-span location of the airfoil. Figure 1 presents a point-cloud acquired during the tests represented as a water-tight surface stereolithography (.stl) file.

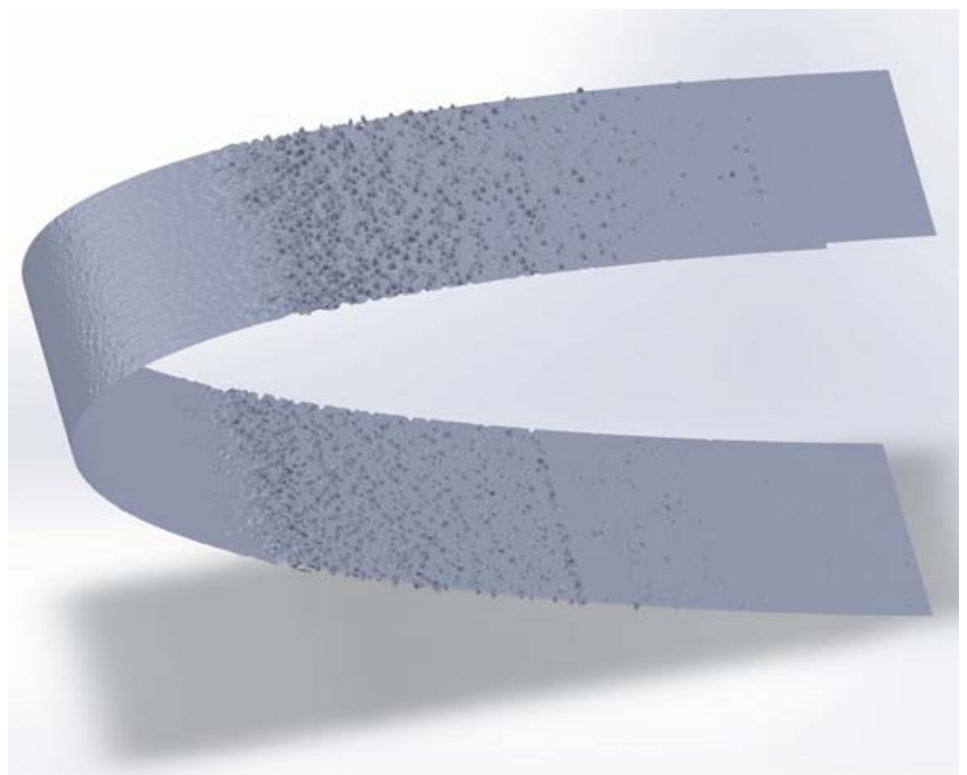

Figure 1. Point Cloud and Water-Tight Surface Representation of the 113012.4 Case

\section{Ice Roughness Characterization}

The ice shape point clouds were processed using the Self-Organizing Map (SOM) approach developed by McClain and Kreeger [9]. The self-organizing map, or sometimes referred to as a Kohonen Map, is a clustering method for the detection of non-linear manifolds, which may be curves or a surfaces, in multi-dimensional space [10]. SOMs depend on the use of codebook vectors, $\mathbf{b}$, which may also be called codebook points or neurons, to represent clumps of data. A set of codebook vectors representing clumps of data points is depicted in on the left side of Figure 2. In its simplest essence, self-organizing maps are employed to capture trends of large data sets by representing those large data set by a relatively small set of codebook vectors.

To capture the trends of a large data set, an SOM algorithm begins by distributing a number of codebook vectors randomly through the space contained by the data set. The codebook vectors are then sequentially moved in the direction of the clump of data points that are closest to the codebook vector. Like most neural network approaches, the SOM requires a learning or training process. Over iterative moves, the codebook vectors spread out and settle into their local clumps. When a series of codebook vectors are connected in a sequence, as shown on the right side of Figure 2, the representative manifold, or curve as is the case in Figure 2, guiding the data through the data space is represented by the path or daisy-chain through the codebook vectors. For more detailed information on selforganizing maps and their application in ice shape description and roughness evaluation, please consult Refs. [8] and [9].

When applied to an iced airfoil or wing without sweep or significant spanwise shape changes, the SOM is expected to identify a curve in the Chord-Chord Normal plane (or x-y plane when the spanwise axis is placed in the z-direction), which represents the mean shape of the iced airfoil. To demonstrate the SOM characterization of an iced airfoil, Figure 3 presents an example point cloud and SOM representation used in the study. The surface 
presented in Figure 3 is the 112912.07 case which exhibited the largest roughness and the highest accumulation parameter (0.3057) measured in this study. Figure 3 demonstrates that the blue codebook vectors capture the shape or curve of iced surface in the projected $x-y$ space.

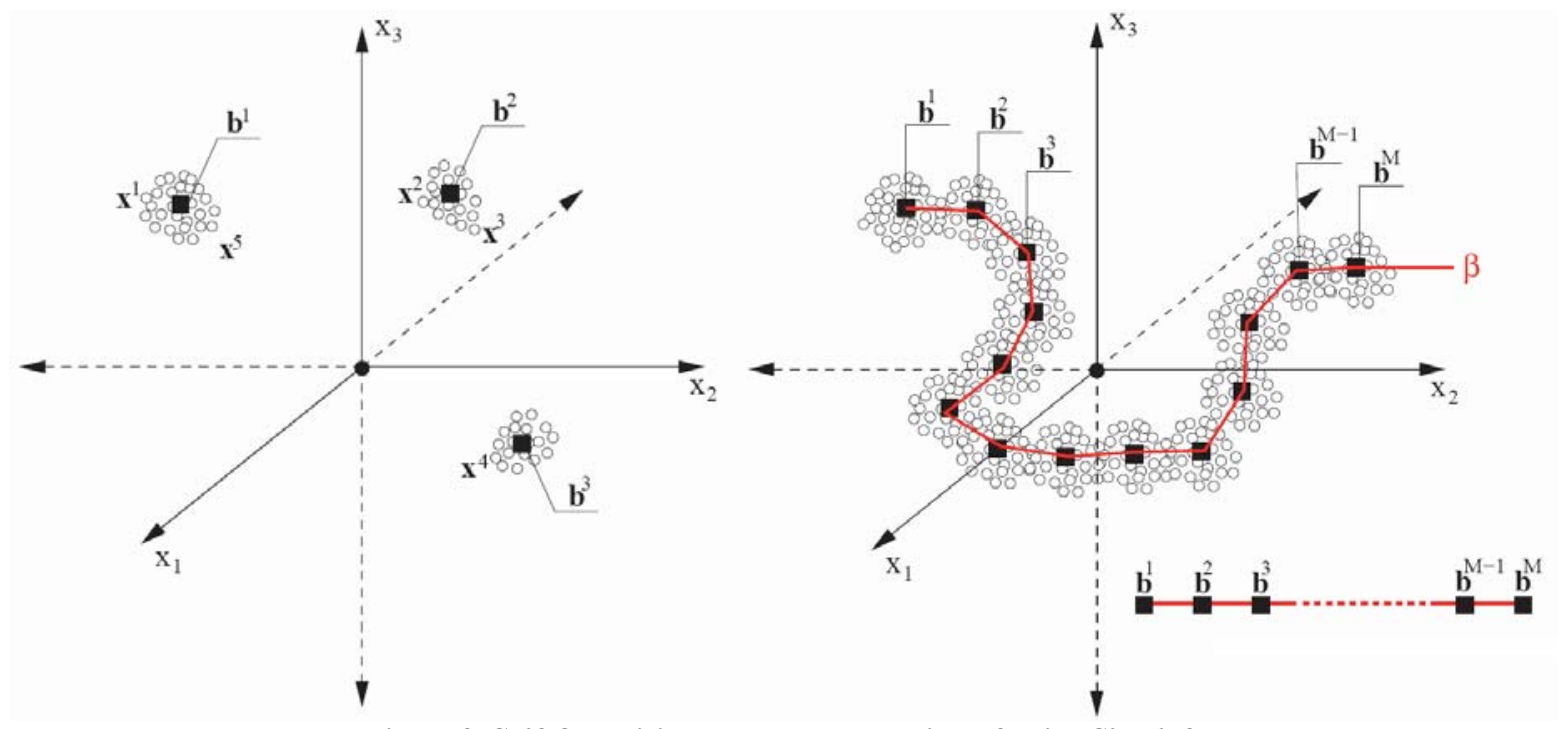

Figure 2. Self-Organizing Map Representation of Point Cloud [9]

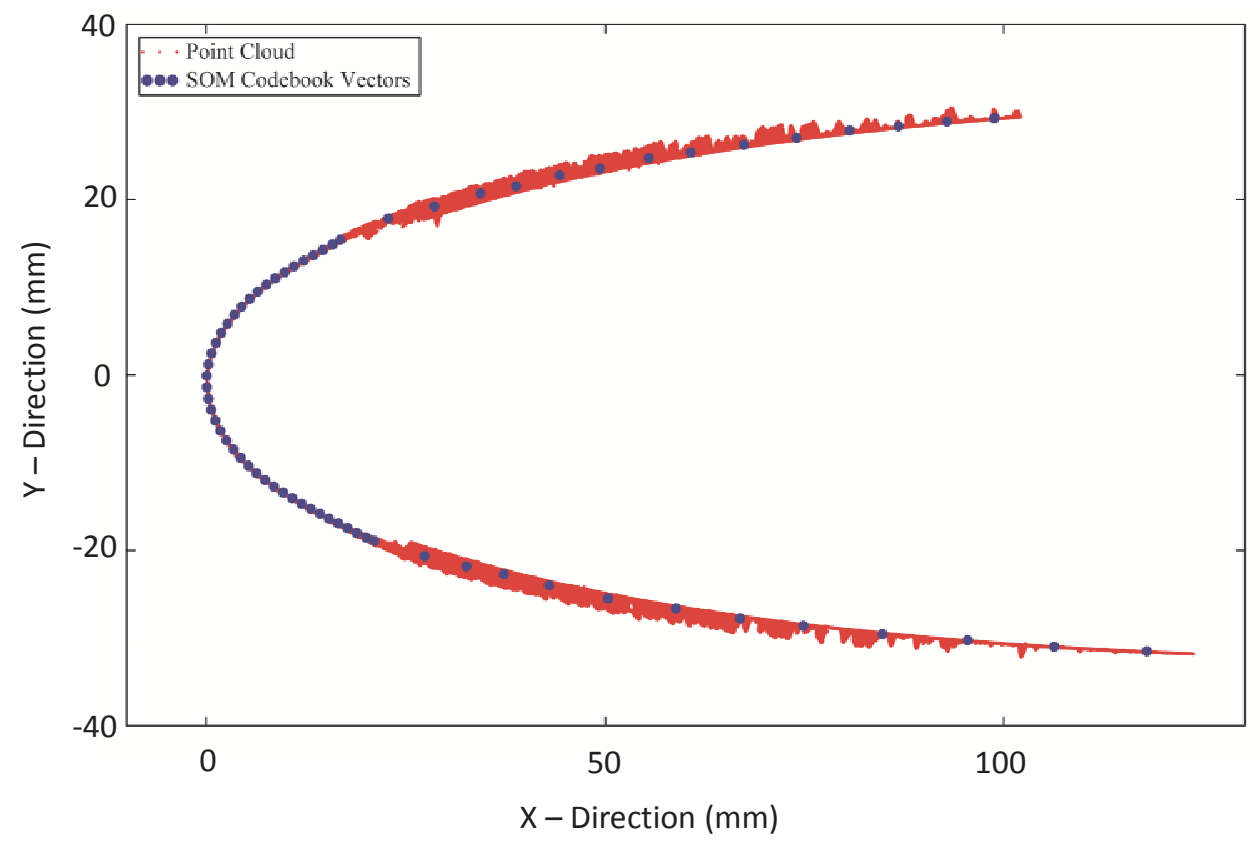

Figure 3. Point Cloud and SOM Representation of 112912.07 Case $\left(M V D=200 \mathrm{um}, \mathrm{LWC}=0.75 \mathrm{gm} / \mathrm{m}^{3}\right.$, and $\Delta t_{s}$ = 94 sec.) which Exhibited the Largest Roughness Values

Figure 3 also demonstrates that different densities of codebook vectors were used in different regions of the airfoil to capture the surface. Following the method presented by McClain and Kreeger [9], a separate SOM characterization with a spacing between $1 \mathrm{~mm}$ and $2 \mathrm{~mm}$ was used to represent the smooth section near the leading edge of the iced airfoil. Further away from the leading edge, the spacing between the codebook vectors was between $6 \mathrm{~mm}$ and $10 \mathrm{~mm}$ in arc length along the surface. Further, Figure 3 demonstrates that while a significant 
amount of ice and roughness was allowed to accumulate on the NACA 0012 airfoil, the ice did not significantly change the apparent aerodynamic shape of the airfoil.

The nature of the SOM method and the positioning of the codebook vectors along a "daisy-chain" enable a statistical evaluation of iced airfoil surface roughness. Since the "clumps" of points are distributed about the codebook vectors, the deviations of the point measurements in the clumps can be used to evaluate the coverage statistics and uncertainty of the codebook vector representation. Figure 4 demonstrates how each surface measurement is used to determine a deviation from the spline surface through the control points or codebook vectors.

Figure 4 shows a single surface measurement $\mathbf{x}^{\mathrm{j}}$, its closest codebook vector $\mathbf{b}^{\mathbf{n}}$. The two neighboring codebook vectors along the daisy-chain of codebook vectors representing the manifold are also shown. In the approach used for this study, the manifold is assumed to be a first-order manifold in two-dimensional space with the characteristic that at each codebook vector, the local slope of the manifold is equal to the central finite-difference evaluated using the two closest surrounding codebook vectors. The approach used assumes that all deviations from the manifold are normal to the manifold. That is, the deviation of a surface measurement normal to the line through the codebook vector with the local slope set by the neighboring codebook vectors is considered the "height" of the surface point above or below the local manifold.

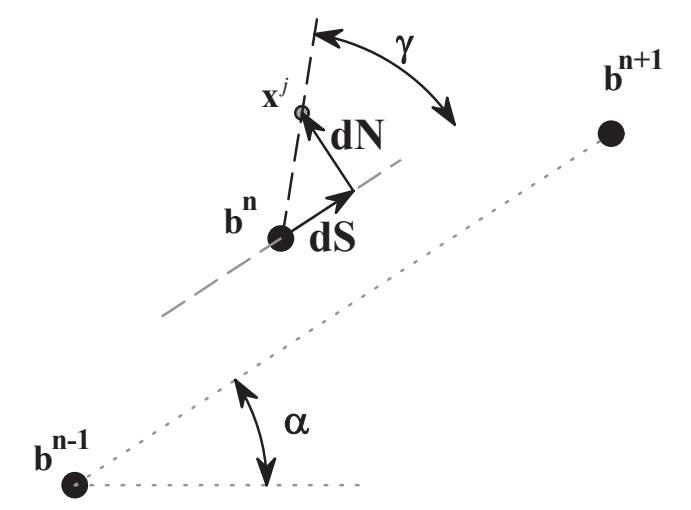

Figure 4. Metrics of Local Point about a Codebook Vector

To calculate the local height, of any point, $\mathbf{x}^{\mathrm{j}}$, relative to the manifold, the two neighboring codebook vectors are first used to calculate the direction of the manifold through $\mathbf{b}^{\mathbf{n}}$ using

$$
\alpha_{\mathbf{b}^{\mathbf{n}}}=\arctan \left[\frac{y_{\mathbf{b}^{\mathrm{n}+1}}-y_{\mathbf{b}^{\mathrm{n}-1}}}{x_{\mathbf{b}^{\mathrm{n}+1}}-x_{\mathbf{b}^{\mathrm{n}-1}}}\right]
$$

The direction of the $\mathbf{x}^{\mathrm{j}}$ point from its winning codebook vector relative to the line through the codebook vector with the direction $\alpha$ is then found using

$$
\gamma_{\mathbf{x}^{j}}=\arctan \left[\frac{y_{\mathbf{x}^{j}}-y_{\mathbf{b}^{\mathbf{n}}}}{x_{\mathbf{x}^{j}}-x_{\mathbf{b}^{\mathbf{n}}}}\right]-\alpha_{\mathbf{b}^{\mathbf{n}}}
$$

The normal height the $\mathbf{x}^{\mathrm{j}}$ point from the line through its winning codebook vector is then determined using

$$
d N_{\mathbf{x}^{j}}=\left[\left(x_{\mathbf{x}^{j}}-x_{\mathbf{b}^{\mathbf{n}}}\right)^{2}+\left(y_{\mathbf{x}^{j}}-y_{\mathbf{b}^{\mathbf{n}}}\right)^{2}\right]^{\frac{1}{2}} \sin \left(\gamma_{\mathbf{x}^{j}}\right)
$$

The normal height of all the points related to an individual codebook vector may then be used to calculate statistics such as those commonly used to calculate traditional roughness parameters. For example, the root-meansquare roughness height for a rough surface is traditionally described as

$$
R_{q}=\left[\frac{1}{J} \sum_{j=1}^{J}\left(y_{j}-\bar{y}\right)^{2}\right]^{\frac{1}{2}}
$$


Based on the SOM-manifold description used here, the root-mean-square roughness height is calculated at each codebook vector as

$$
R_{q}=\left[\frac{1}{J} \sum_{j=1}^{J} N_{\mathbf{x}^{j}}{ }^{2}\right]^{\frac{1}{2}}
$$

In traditional roughness studies, the $99.9 \%$ roughness maximum height (RMH) based on a Gaussian distribution is calculated using 3.09 times the root-mean roughness height.

$$
R M H_{\mathbf{b}^{\mathbf{n}}}=3.09 R_{q}==3.09\left[\frac{1}{J} \sum_{j=1}^{J} N_{\mathbf{x}^{j}}\right]^{\frac{1}{2}}
$$

Where $J$ is the number of surface points for which $\mathbf{b}^{\mathbf{n}}$ is the winning (closest) codebook vector. The RMH is the local 99.9\%-maximum roughness height and is evaluated at each codebook vector based on the local or neighborhood statistics. The RMH will vary along the surface arc length.

To evaluate the distance along the manifold representing the mean ice shape, a discrete arc-length approach is taken. That is, at one end of the daisy chain, the length of the arc is set to zero or a known value from the stagnation point on the airfoil. The position of the next codebook vector is then evaluated as the straight-line distance between the two codebook vectors as demonstrated in Eq. (7).

$$
S_{\mathbf{b}^{\mathbf{n}}}=S_{\mathbf{b}^{\mathbf{n}-1}}+\left[\left(x_{\mathbf{b}^{\mathbf{n}}}-x_{\mathbf{b}^{\mathrm{n}-1}}\right)^{2}+\left(y_{\mathbf{b}^{\mathbf{n}}}-y_{\mathbf{b}^{\mathbf{n}-1}}\right)^{2}\right]^{\frac{1}{2}}
$$

Once the surface distance coordinate of each codebook vector is determined, the surface distance coordinate of each point cloud measurement may be evaluated based on the location of the surface point's winning codebook vector. Revisiting Figure 3, once the angle of the $\mathbf{x}^{\mathrm{j}}$ point with respect to the surface manifold through its winning codebook vector $(\gamma)$ is known, the surface projection along the manifold is found using

$$
S_{\mathbf{x}^{j}}=S_{\mathbf{b}^{\mathbf{n}}}+d S_{x^{j}}=S_{\mathbf{b}^{\mathbf{n}}}+\left[\left(x_{\mathbf{x}^{j}}-x_{\mathbf{b}^{\mathbf{n}}}\right)^{2}+\left(y_{\mathbf{x}^{j}}-y_{\mathbf{b}^{\mathbf{n}}}\right)^{2}\right]^{\frac{1}{2}} \cos \left(\gamma_{\mathbf{x}^{j}}\right)
$$

The straight-line arc length approach of Eq. (7) is not the only option for evaluating the length of the manifold between the codebook vectors. An approach that connects the codebook vectors using the lines through the codebook vectors with the directions and slopes evaluated using Eq. (1) may be a more appropriate method than that presented in Eq. (7). However, the salient features of any unwrapping process are captured by the method employing Eqns. (7) and (8). Those critical features being 1) the distance between the codebook vectors along the manifold are calculated and then 2) the projected distance of each surface point from its winning codebook vector is calculated relative to the directions tangent to and normal to the manifold passing through the winning codebook vector.

\section{Roughness Measurement Results}

The specific aims of the study were to 1) characterize how SLD roughness develops with increasing accumulation parameter given fixed cloud properties and 2) characterize the influence of the MVD on ice roughness provided equivalent accumulation parameters. In the following sections, each aim is discussed separately.

\section{Accumulation Parameter (Icing Event Duration) Investigation}

Each of the ice shape scans described in Table 1 consists of more than one million surface points. Consequently, reducing the point clouds and understanding the implications of the reduced roughness data is inappropriate without an appreciation for the rough surface topographies captured in the study. Figure 5 presents a scatter plot of the unwrapped surface topographies for four of the $200-\mu \mathrm{m}$ cases. Since a two-dimensional SOM approach was used to characterize the point clouds, the spanwise (z) coordinate of each point in the original point cloud was not modified for a given scan. Approximately $20-\mathrm{mm}$ strips from the spanwise center of the scan were extracted for Figure 5, and points only on one side of the airfoil were plotted (i.e. the "0" distance point on the x-axis represents the stagnation point of the iced airfoil). 
The color map for Figure 5 was set based on the elevation values of the 94-sec. case to provide a consistent comparison of the accretion time-development of roughness. The elevation changes shown in Figure 5(a) for the 27 -sec. case are barely noticeable given the scaling mandated by the $94-\mathrm{sec}$. case. Because of its very small roughness values and because of the lack of resolution for the 27 -sec. case, the 19-sec accretion case at $200 \mu \mathrm{m}$ and $0.75 \mathrm{gm} / \mathrm{m}^{3}$ (Run 112912.08) was omitted from Figure 5. As the accretion times grow and the accumulation parameter increases, the surface features become more and more pronounced in Figure 5.
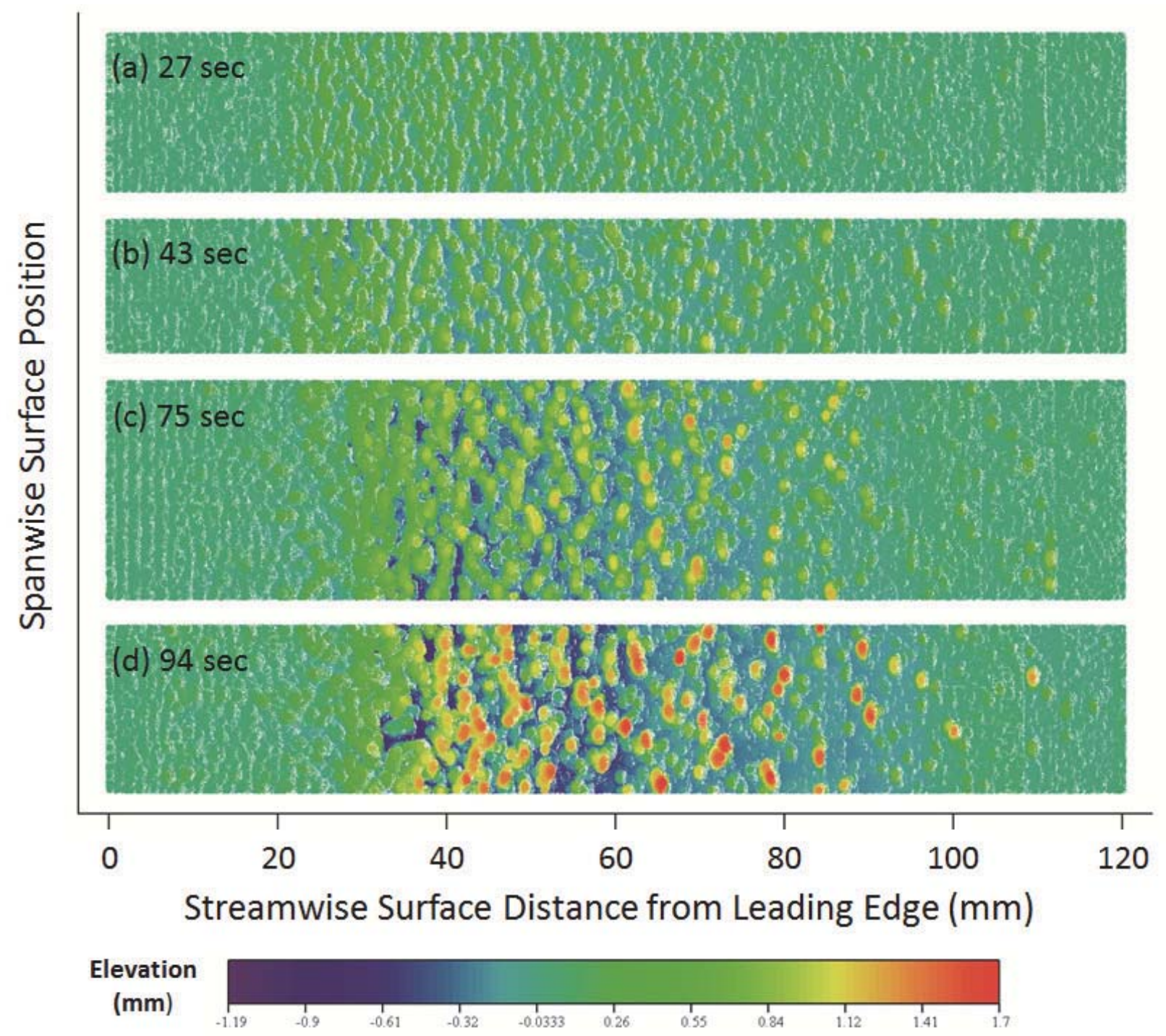

Figure 5. Surface Topography Visualization along the Airfoil Surface for the 200-um, $0.75 \mathrm{gm} / \mathrm{m}^{3}$ Cases

Figure 6, 7, and 8 present the maximum roughness height (RMH) measurements, calculated as three times the root-mean-square roughness height, along the surfaces for the $100-\mu \mathrm{m}, 150-\mu \mathrm{m}$, and $200-\mu \mathrm{m}$ cases, respectively. In Figures 6,7, and 8, the "zero" mean shape surface distance represents the location of the stagnation point on the iced airfoil. Positive values of mean shape surface distance represent surface distance variations along the right side of the airfoil when an observer is aligned with the spanwise axis of the airfoil and is looking into the direction of the oncoming flow. Negative values represent surface distance along the left side of the airfoil. Figures 6, 7, and 8 all show that the roughness values along the leading edge-region become more and more rough with increasing ice accretion time or accumulation parameter.

However, Figures 6, 7, and 8 present some issues in quantifying the variation in ice roughness with accretion time. First, there is not a defined region where the RMH values are constant in the direction of the flow. The magnitude of the RMH varies continuously along the surface. Secondly, Figures 6-8 demonstrate that the roughness 
variations are not symmetric even though all of the cases were run for a symmetric airfoil at $0^{\circ}$ AOA. The value of roughness at a location on the right ice surface generally does not equal the roughness at the reflected location on the left ice surface of the airfoil. Finally, in investigating the roughness variations with accretion time, the location of the roughness comparison matters. For example in Figure 6, the surface maximum RMH exhibited by the 100- $\mu$ m, 75 -sec. case is actually larger than the $100-\mu \mathrm{m}, 94$-sec. case. However, at $\pm 60 \mathrm{~mm}$ from the leading edge, roughness values appear to progress proportionally to the ice accretion time.
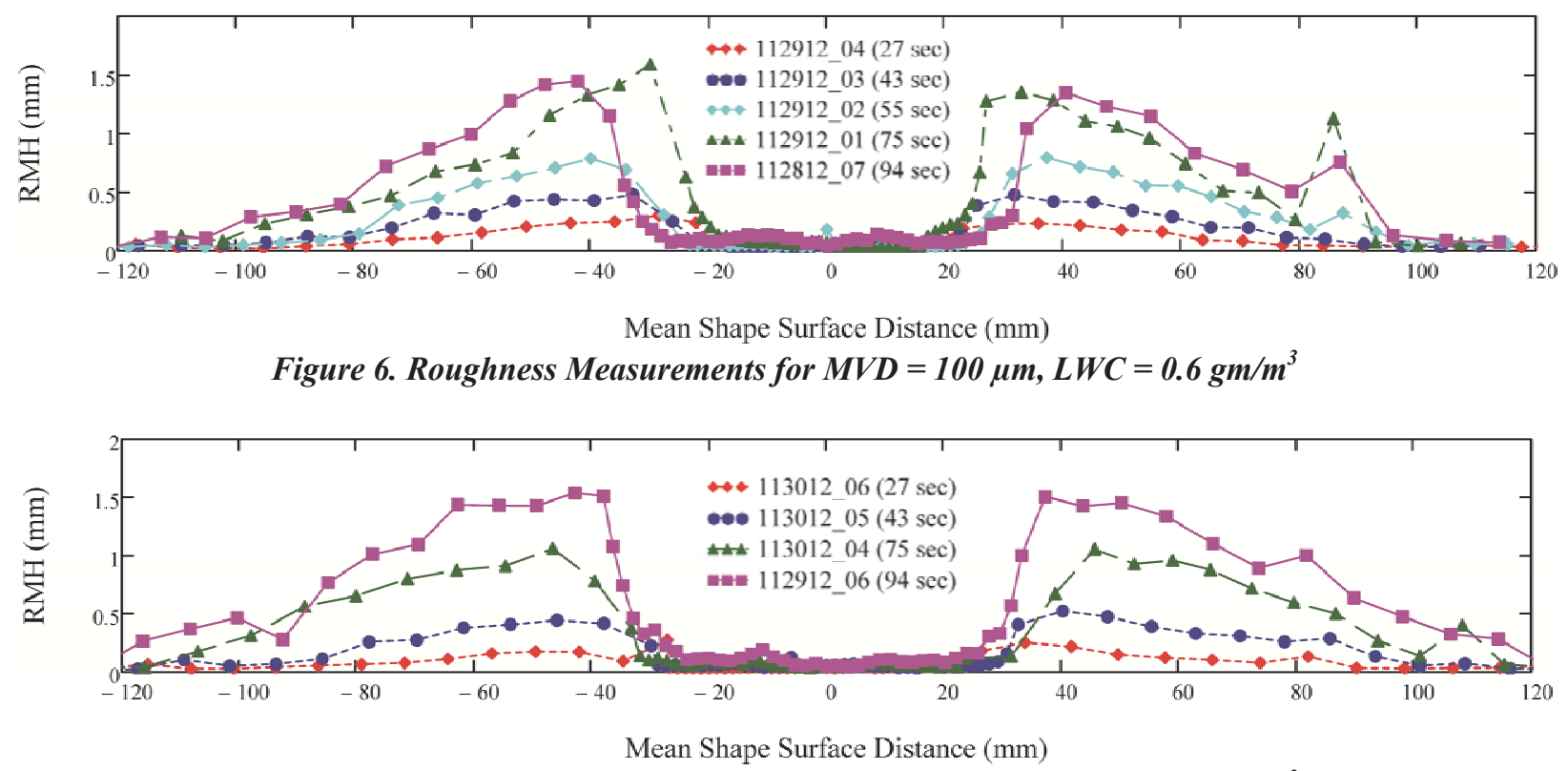

Figure 7. Roughness Measurements for $M V D=150 \mu \mathrm{m}, L W C=0.6 \mathrm{gm} / \mathrm{m}^{3}$

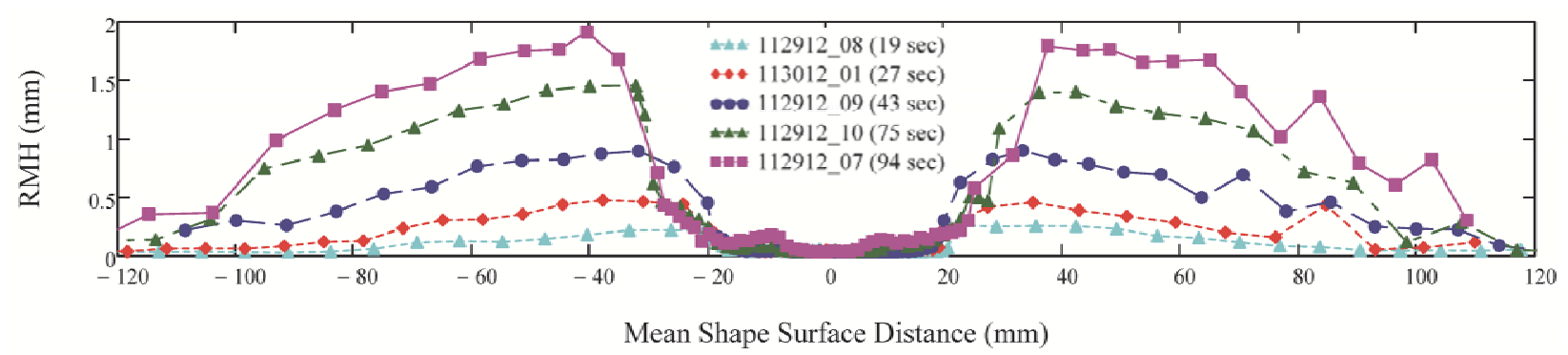

Figure 8. Roughness Measurements for $M V D=200 \mu \mathrm{m}, L W C=0.75 \mathrm{gm} / \mathrm{m}^{3}$

Given the asymmetry and surface roughness variations for each case, the SLD cases were compared in their development versus accretion parameter using the average-maximum RMH. That is, for a given experimental test case, the maximum local roughness height on the right side was averaged with the maximum local roughness height on the left side of the airfoil.

$$
R M H_{\max }=\frac{R M H_{\max , \text { Right }}+R M H_{\max , \text { Left }}}{2}
$$

Figure 9 presents the airfoil $R M H_{\max }$ values as a function of accumulation parameter for the SLD cases. Figure 9 demonstrates a linear relationship between the airfoil maximum roughness height and the accumulation parameter. Interestingly, only one data point is an outlier with a deviation of $0.4 \mathrm{~mm}$ from the predicted maximum RMH, while two of the 100 um cases (the $A_{c}=0.0703$ and $A_{c}=0.1120$ cases) are not visible in Figure 9 because they are covered by blue circles of the 150 micron cases. 


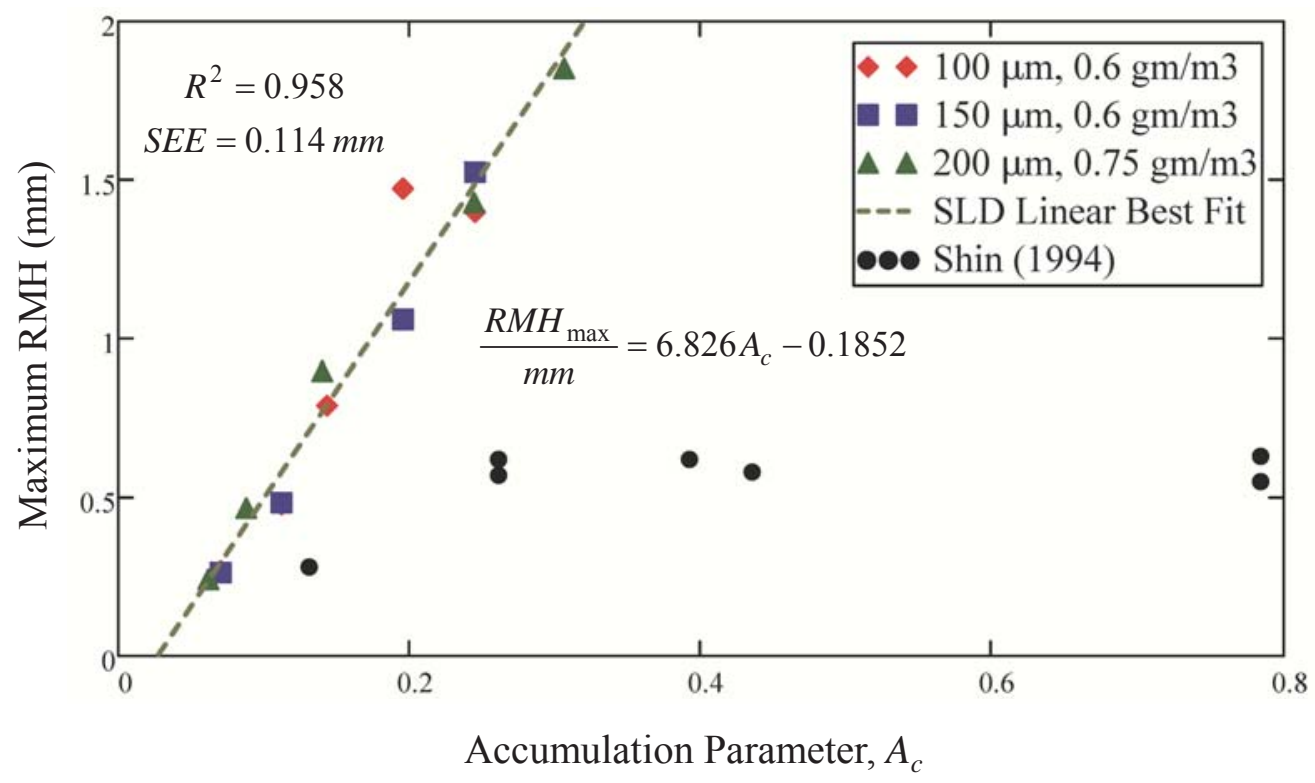

Figure 9. Airfoil Maximum Roughness Height as a Function of Accumulation Parameter

The results from Figure 9 differ from the findings of Shin [3] who studied Appendix "C" roughness on a 53.3 $\mathrm{cm}\left(21 \mathrm{in}\right.$.) NACA 0012. Shin [3] exposed the NACA 0012 to clouds with MVD $=20 \mu \mathrm{m}$ and $\mathrm{LWC}=0.5 \mathrm{gm} / \mathrm{m}^{3}$ and with velocities of $67.1 \mathrm{~m} / \mathrm{s}$ or $111.8 \mathrm{~m} / \mathrm{s}$. Images taken looking down the spanwise axis at the surface of the airfoil were then used to measure the roughness element heights. The data reported in Shin [3] are included in Figure 9 and demonstrate that while an increase in roughness levels occurred at low accumulation parameters, the "Appendix C" roughness measurements plateaued at $0.6 \mathrm{~mm}$. Further, the roughness heights increased less quickly in the study of Shin [3] than in the current SLD study.

Many factors are likely contributors to the difference in the rates of roughness growth and the limits to roughness growth noted by Shin [3] and those found in this study. The most significant difference between the measurement of Shin [3] and those of this study is the significant difference in the MVD's explored. Further research using laser scanning of "Appendix C" ice roughness is required to determine whether or not the MVD's employed caused the difference between the ice roughness behaviors. Other suspects for the difference between the measured roughness growth rates include 1) the difficulties in performing image analysis when observing small surface features from long distances using a zoom lens, 2) the arbitrary nature of picking out "representative" roughness elements from a field, and 3) the lack of detail in the Shin [3] study of where, in terms of distance from the stagnation point, the roughness element was measured.

\section{MVD Comparison}

In Figure 9, two accumulation parameters exist with measurements made for each of the three MVD values: $A_{c}$ $\approx 0.244$ and $A_{c} \approx 0.070$. Studying the cases at these two accumulation parameters provide a deeper investigation into the influence of the MVD on ice roughness statistics and topography.

Figure 10 presents the local RMH variations along the airfoil surfaces for each of the three MVD cases for the lower accumulation parameter $(\approx 0.070)$. Figure 10 shows that there are differences between the three cases at the lower accumulation parameter. That is, there is some separation between the three cases especially on the left-side of the airfoil. However, when the average values between the left and right side are compared, especially at the maximum values presented in Figure 9, the differences between the cases becomes negligible.

Figure 11 presents the local RMH variations along the airfoil surfaces for the higher accumulation parameter $(\approx$ 0.244). Figure 11 along with Figure 9 show that when comparing the surface maximum RMH values, the maximum RMH values are essentially independent of the MVD. However, Figure 11 suggests that downstream of the location of the surface maximum RMH values, variations in surface roughness statistics are affected by MVD. In Figure 11, the $100-\mu \mathrm{m}$ case is generally less rough than the other cases downstream of the point of local maximum RMH values. While a quantitative assessment is not provided, the smooth region also appears larger for the $100-\mu \mathrm{m}$ case than for the other two cases. 


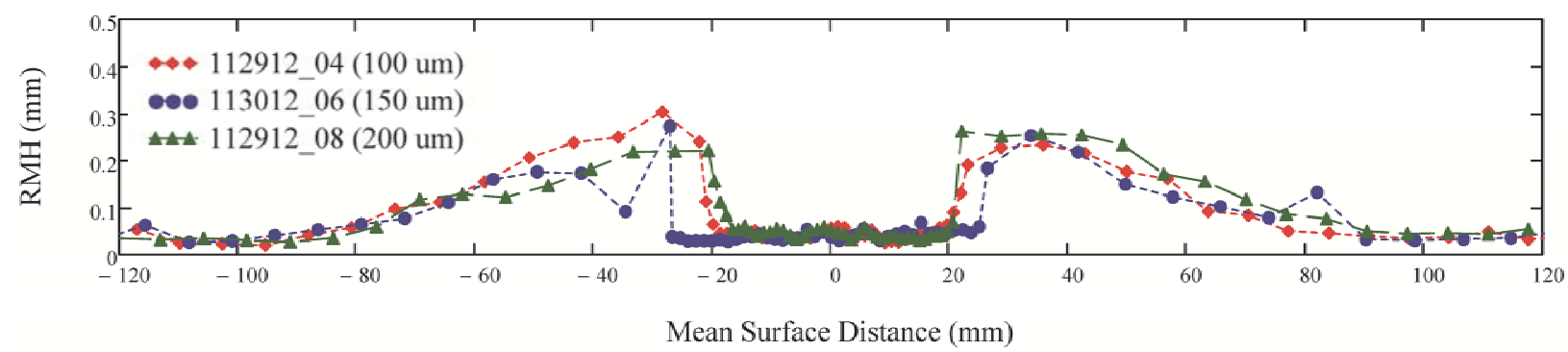

Figure 10. Roughness Comparison for an Accumulation Parameter of Approximately 0.070 (Shorter Event)

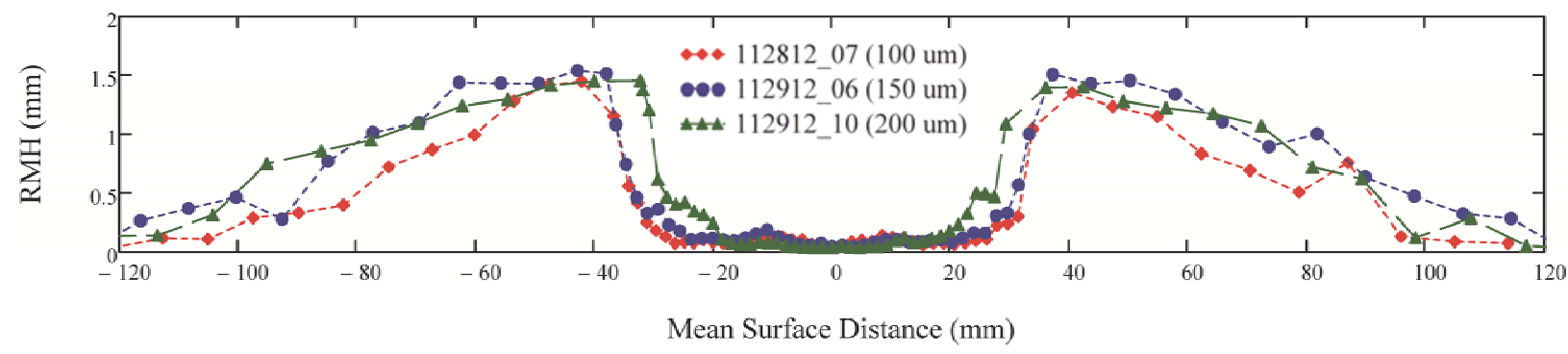

Figure 11. Roughness Comparison for an Accumulation Parameter of Approximately 0.244 (Longer Event)

Figure 12 presents the topographies of the 0.244 accumulation parameter cases. While the maximum RMH values are very similar, Figure 12 reiterates the findings from Figure 11: for the $100-\mu \mathrm{m}$ case, the roughness starts further downstream and decays faster than for the other two cases.

\section{Conclusions}

A series of experiments was performed to quantify the development of roughness on iced airfoils during supercooled large droplet (SLD) icing. The measurements were performed on a straight NACA 0012 at $0^{\circ}$ AOA and at conditions matching historical studies of "Appendix C" icing roughness. The resulting ice shapes were scanned and analyzed using the method of McClain and Kreeger [9] for roughness evaluation. The primary findings of the study were:

1. Ice roughness varies continuously along the iced surface of the airfoil. Because of the continuously varying properties, comparing ice accretion roughness must be performed using the airfoil maximum values or at a specific distance along the iced airfoil surface.

2. For the three MVD's chosen for the study, the airfoil maximum ice roughness increased proportionally to the accretion time or accumulation parameter. This finding deviates from historical Appendix C studies which found that roughness height became capped at around $0.6 \mathrm{~mm}$ as the accumulation parameters increased.

3. While the airfoil maximum roughness was found to vary primarily with accumulation parameter, the cloud MVD influenced the roughness variation along the surface of the airfoil. As the MVD decreased and for the longer accumulation parameter studied, the roughness region started further from the leading edge and decayed faster as the distance from the leading edge increased.

The results of the study provide significant insights into the development of roughness during ice accretion on airframe surfaces in SLD conditions. The new insights also represent a substantial resource for modeling the SLD ice accretion process using codes such as LEWICE. 


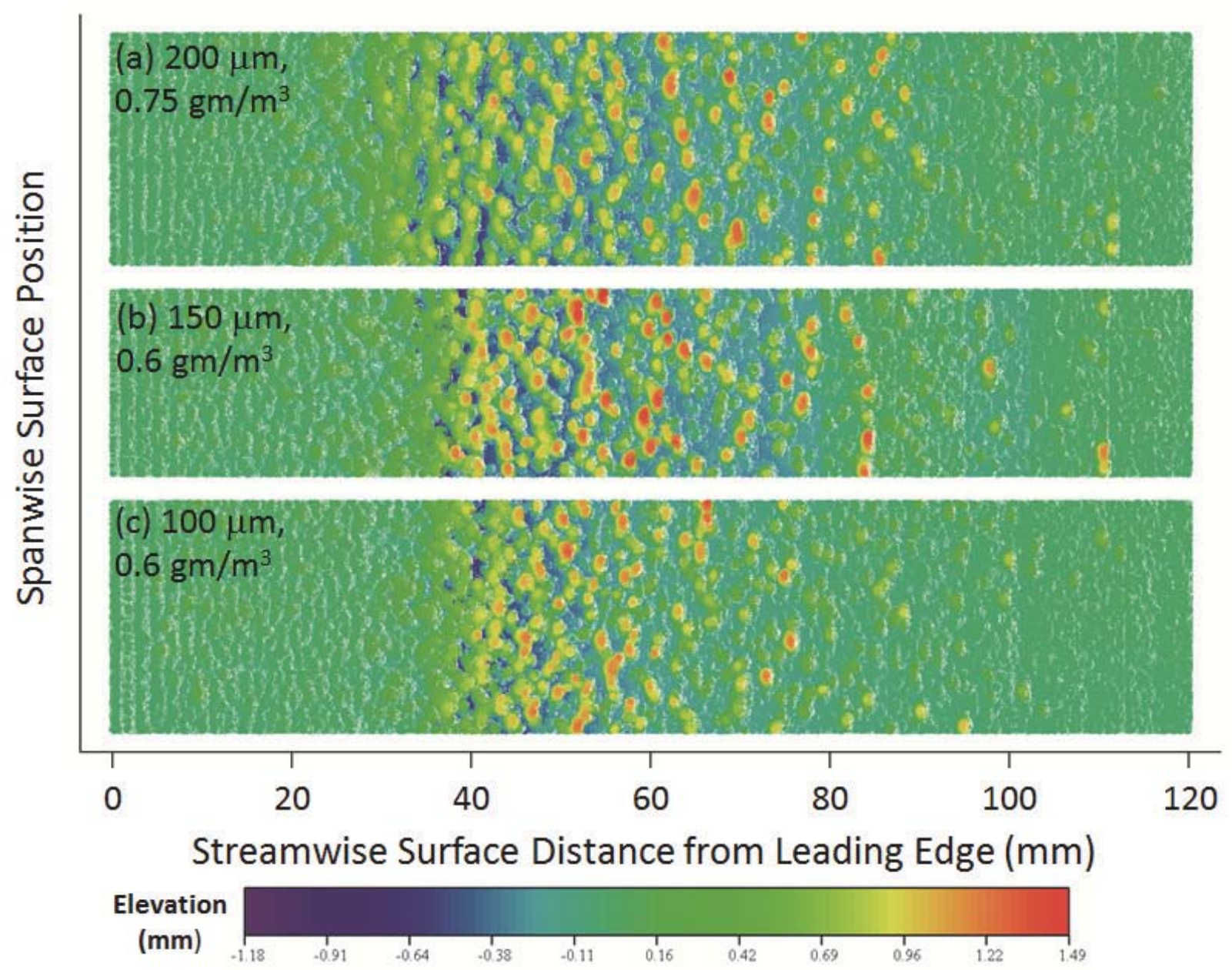

Figure 12. Topographical Comparison of Surface Features for Cases with an Accumulation Parameter of 0.244

\section{Acknowledgments}

The efforts reported in this paper were partially supported as part of NASA Collaborative Agreement No. NNX12AB85A. The authors thank Drs. Sam Lee and Andy Broeren for their assistance with the study. Finally, the authors thank Peter Tino of the University of Birmingham, UK whose class notes inspired the initial SOM investigation of ice shapes and who provided many suggestions for improving the roughness characterization approach in the early stages if its development. Any opinions presented in this paper are those of the authors and do not reflect the views of NASA or the United States government.

\section{References}

${ }^{1}$ Anderson, D. N., and Shin, J., (1997), "Characterization of Ice Roughness from Simulated Icing Encounters," NASA TM-107400.

${ }^{2}$ Anderson, D. N., Hentschel, D. B., and Ruff, G. A., (1998), "Measurement and Correlation of Ice Accretion Roughness," NASA CR-2003-211823.

${ }^{3}$ Shin, J., (1994), "Characteristics of Surface Roughness Associated With Leading Edge Ice Accretion," NASA TM-106459.

4"14 CFR Part 25: Airworthiness Standards: Transport Category Airplanes - Appendix C, Part I: Atmospheric Icing Conditions," (2012), Code of Federal Regulations.

5"14 CFR Parts 25 and 33: Airplane and Engine Certification Requirements in Supercooled Large Drop, Mixed Phase, and Ice Crystal Icing Conditions," (2010), Federal Register, Vol. 75, No. 124, June 29, 37311-37339.

${ }^{6}$ Wright, W.B., "User Manual for the NASA Glenn Ice Accretion Code LEWICE," NASA/CR-2002-211793, 2002. 
${ }^{7}$ Lee, S., Broeren, A., Addy, H., Sills, R., and Pifer, E., (2012), "Development of 3D Ice Accretion Measurement Method," AIAA-2012-2938, presented at the $4^{\text {th }}$ AIAA Atmospheric and Space Environments Conference, June 2528.

${ }^{8}$ McClain, S. T., Tino, T., and Kreeger, R. E., (2011), “Ice Shape Characterization Using Self-Organizing Maps," Journal of Aircraft, Vol. 48, No. 2, March-April.

${ }^{9}$ McClain, S. T. and Kreeger, R. E., (2013), "Assessment of Ice Shape Roughness Using a Self-Organizing Map Approach," Presented at the 5th AIAA Atmospheric and Space Environments Conference, June 24-27, San Diego, CA, AIAA-2013-2546.

${ }^{10}$ Kohonen, T., (2001), Self-Organizing Maps, Berlin: Springer-Verlag, 3rd ed. 